\title{
A small satellite mission for solar coronagraphy
}

\section{N. Gopalswamy, Qian Gong}

N. Gopalswamy, Qian Gong, "A small satellite mission for solar coronagraphy," Proc. SPIE 10769, CubeSats and NanoSats for Remote Sensing II, 107690X (18 September 2018); doi: 10.1117/12.2323807

SPIE Event: SPIE Optical Engineering + Applications, 2018, San Diego, California, United States 


\title{
A small satellite mission for solar coronagraphy
}

\author{
N. Gopalswamy*a, Qian Gong ${ }^{\mathrm{b}}$

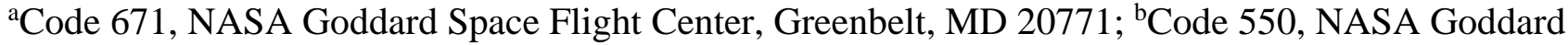 \\ Space Flight Center, Greenbelt, MD 20771
}

\begin{abstract}
We present on a concept study of the Goddard Miniature Coronagraph (GMC) mission for measuring the plasma flow in the solar corona in the form of solar wind and coronal mass ejections (CMEs). These mass flows can dramatically alter the near-Earth space environment to hazardous conditions posing danger to human technology in space. The primary science objective of the mission is to measure the properties of CMEs, coronal structures, and the solar wind near the Sun. The miniaturization of the coronagraph involves using a single-stage optics and a polarization camera, both of which reduce the size of the coronagraph. GMC will be accommodated in a small satellite that can be built with cubesat material to minimize cost. The development of the Dellingr mission at NASA/GSFC has provided expertise and a clear pathway to build the GMC mission. The hardware and software used for the Dellingr mission are technically sound, so the GMC mission can be fully defined. Software, pointing, control and communications systems developed for GSFC CubeSats can be readily adapted to cut costs. We present orbit options such as an ISS orbit or a Sun synchronous dawndusk polar orbit with the aim of maximizing solar observations.
\end{abstract}

Keywords: Coronal mass ejections, solar wind, coronagraph, SmallSat mission

\section{INTRODUCTION}

Coronagraphy has become a critical technique in investigating the energy flow from the Sun in the form of coronal mass ejections (CMEs) and solar wind. Understanding how CMEs evolve near the Sun and how the solar wind is accelerated are goals of many investigations in heliophysics. CMEs cause intense geomagnetic storms and large solar energetic particle events. These space weather events have serious consequences to life on Earth and technological systems in space. Currently, coronagraphs on board the ESA/NASA mission Solar and Heliospheric Observatory (SOHO) provide continuous images of the corona since 1996, so CMEs can be observed and tracked ${ }^{1}$. Given the old age ( $>20$ years) of SOHO, heliophysics investigations will suffer when the coronagraphs on board SOHO fail. While the coronagraph on board the Solar Terrestrial Relations Observatory $\left(\right.$ STEREO ${ }^{2}$ ) is highly useful for science, continuous monitoring of CMEs is needed for space weather purposes. The NASA/GSFC coronagraph team has been developing the Goddard Miniature Coronagraph (GMC) that can be quickly built when the need arises. A spacecraft carrying GMC and deployed in a Low Earth Orbit will address the need of the heliophysics community of having a coronagraph to observe solar events without interruption.

In a recent report ${ }^{3}$, the Committee on Achieving Science Goals with CubeSats of the US National Academy of Sciences recommends that solar imagers be packaged into CubeSats. The three imagers in high demand for tracking solar eruptions are the magnetic imager (to obtain magnetograms that show the locations of active regions on the Sun), the EUV imager that images the early phase of CMEs and the coronagraph that images CMEs that propagate into the heliosphere. CMEs take about 1-4 days to reach Earth, so observing their onset near the Sun provides important early warning of their potential impact. The key developments that need to happen to achieve such missions are miniaturization instruments and the development of high-rate communication and pointing capabilities. The Goddard Miniature Coronagraph (GMC) aims at the miniaturization of coronagraphs. This paper describes the results of a concept study to deploy GMC in a Low Earth Orbit as a sole payload on a small satellite.

\section{REQUIRED MEASUREMENTS}

A coronagraph is an instrument that blocks the bright photosphere using an occulting disk so that the faint corona can be imaged. Solar eruptions and the solar wind originate from the corona. Traditionally, the coronagraphs provided density information on the structures in the corona including CMEs. CMEs propagate in the ambient medium, which is the solar wind. While the CMEs attain their peak speed within $\sim 3$ solar radii (Rs), the solar wind just starts picking up around this 

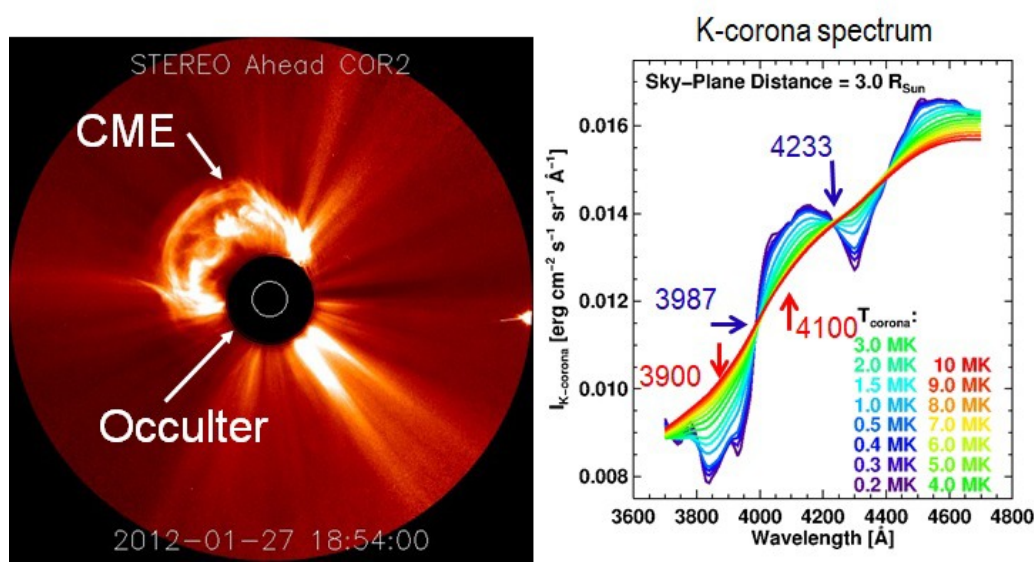

Two passband ratios of interest

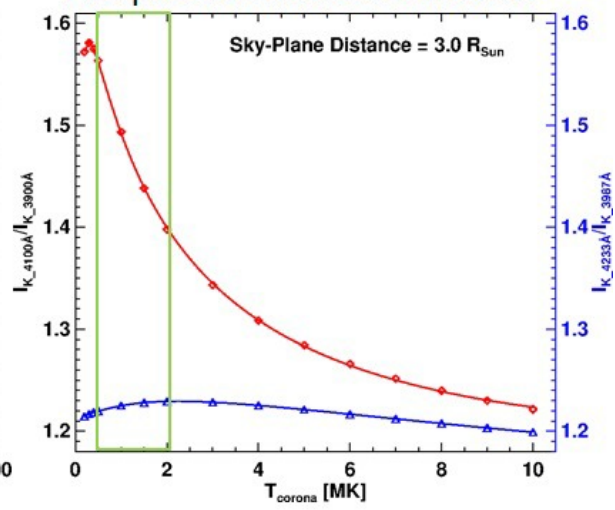



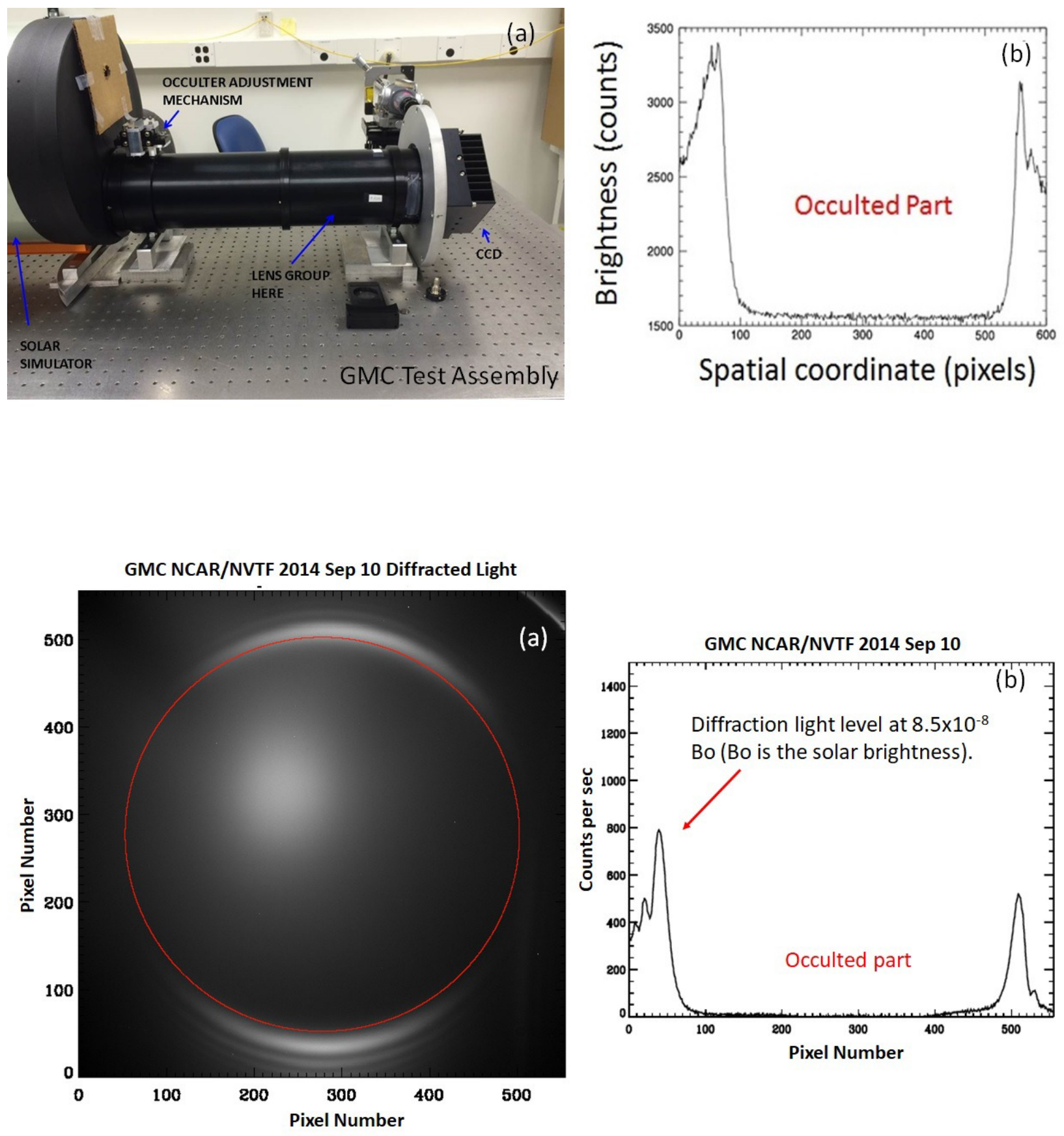
The diffraction pattern is not uniform around the Sun because it was not possible to properly align the IRIS (functions as an aperture stop) is not accurately aligned to the axis formed by the commercial lens group and the occulter. The peak diffracted stray light is at $8.5 \times 10^{-8} \mathrm{Bo}$, similar to the air test results. Note that the diffracted stray light falls off rapidly after the initial peaks. Based on these successful tests, the GMC mission will use the threaded cone occulter design and use a lens group from our own optical design. In addition, the CCD camera used in the test assembly will be replaced by a polarization camera. The coronagraph to be used in the space flight will have only two mechanisms: the aperture door (one-time opening) and a filter-wheel rotator mechanism. This represents one less mechanism compared to the traditional coronagraphs (they typically have a polarization wheel and a filter wheel). The FOV of GMC is similar to that of the SECCHI outer coronagraph COR2 ${ }^{2}$. With an aperture size of $40 \mathrm{~mm}$, the light gathering power of GMC is much larger than that of LASCO C2 $(20 \mathrm{~mm})$ and C3 $(9 \mathrm{~mm})$ coronagraphs, but slightly larger than that of COR2 $(34 \mathrm{~mm})$. Another major improvement is in the use of polarization camera that eliminates the problem of image smear that occurs when a sequence of images obtained from three polarization positions are combined to obtain the polarized and total brightness of the corona. For example, COR2 takes 11 seconds to complete a polarization sequence. Over this period, a fast CME with a speed of $\sim 1500 \mathrm{~km} / \mathrm{s}$ travels a lot more ( 23 arc sec) than the pixel size (14.7 arc sec).

Table 1. The design parameters of the GMC optical assembly

\begin{tabular}{|l|l|}
\hline Parameter & Value \\
\hline FOV (radius from Sun center) & $15 \mathrm{R}_{\mathrm{S}}$ \\
\hline Inner FOV cutoff (from Sun center) & $3 \mathrm{R}_{\mathrm{S}}$ \\
\hline Wavelength $(\lambda$ ) range (four narrowband, 1 broadband) & $380-460 \mathrm{~nm}$ \\
\hline Effective Focal Length & $103 \mathrm{~mm}$ \\
\hline Entrance pupil diameter & $40 \mathrm{~mm}$ \\
\hline Detector array & $\mathrm{CCD}, 1950 \times 1950,7.4 \mu \mathrm{m}$ pixel \\
\hline Throughput (average over $\lambda$ \& FOV) & $>90 \%$ \\
\hline
\end{tabular}

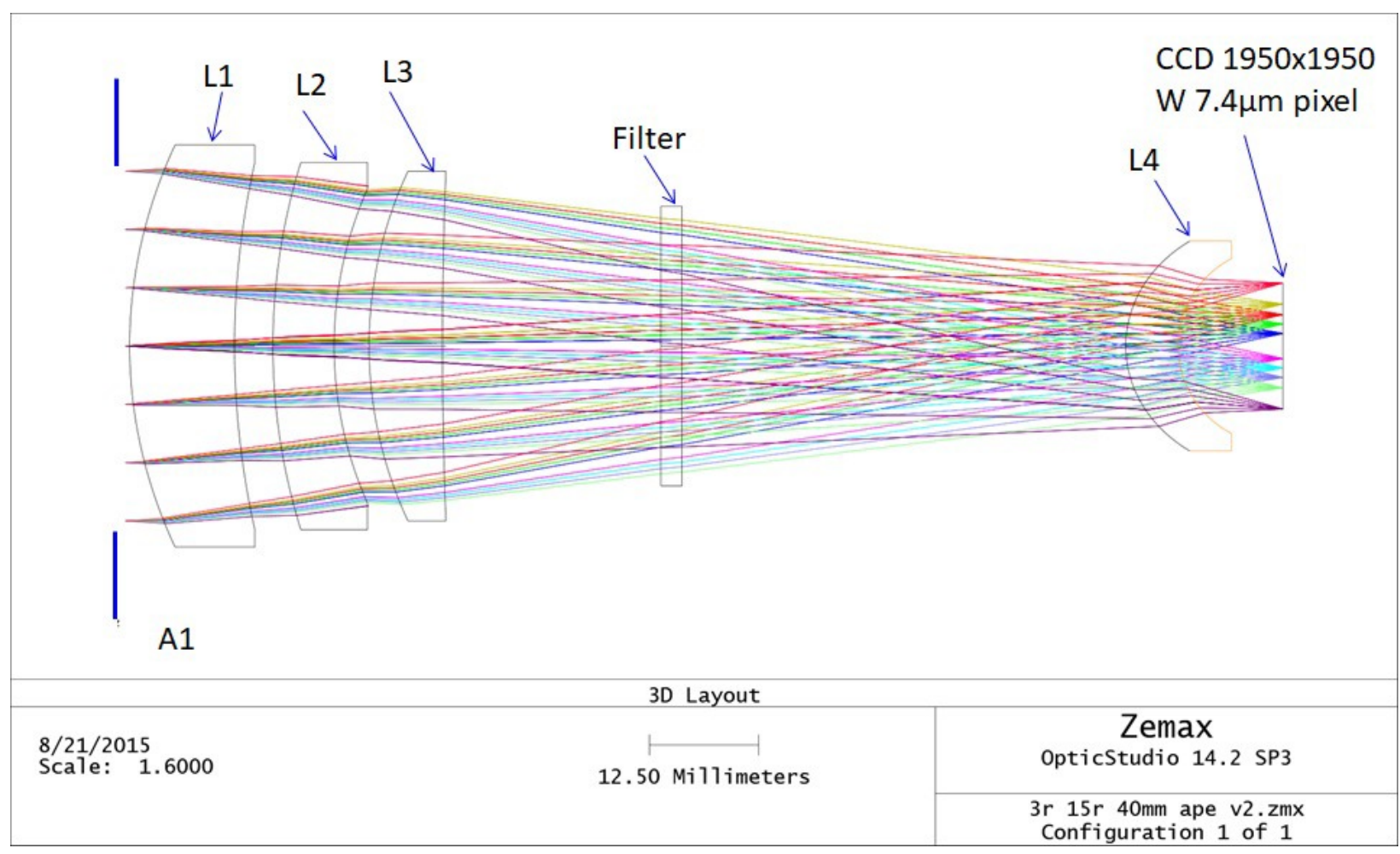

Figure 4. GMC optical design showing the aperture A1, the lens group (L1+L2+L3), the field corrector L4, the filter position, and the focal plane. 


\subsection{Optical design}

The design parameters for GMC are listed in Table 1 have some optimization done with respect to the test setup shown in Fig. 2. The circular FOV has a radius of 15 Rs. The range 3-5 Rs is important for isolating the K-corona by the polarization technique to study the solar wind properties using the passband ratio imaging. The full FOV will be useful for traditional coronagraphy that uses total brightness to obtain CME properties. The wavelength band of the coronagraph is chosen to do passband ratio imaging and traditional coronagraphy. The effective focal length of $103 \mathrm{~mm}$ is optimized so the coronal image fills the $1950 \times 1950$ CCD. The plate scale works out to be $\sim 29$ arcsec per pixel because of the polarization mask used on the CCD requires $2 \times 2$ binning. The throughput includes the loss of the surface reflection of material absorption, but does not include the transmittance of the spectral filters and the polarizer loss. The optics was optimized using the optical design software Zemax and the resulting layout is shown in Fig. 4. Not shown are the external occulter in front of the aperture A1 and the polarization camera in the image plane. Zemax analysis of the image quality (spot diagram) shows that the size of the point spread function is much smaller than the detector pixel size across the full FOV.
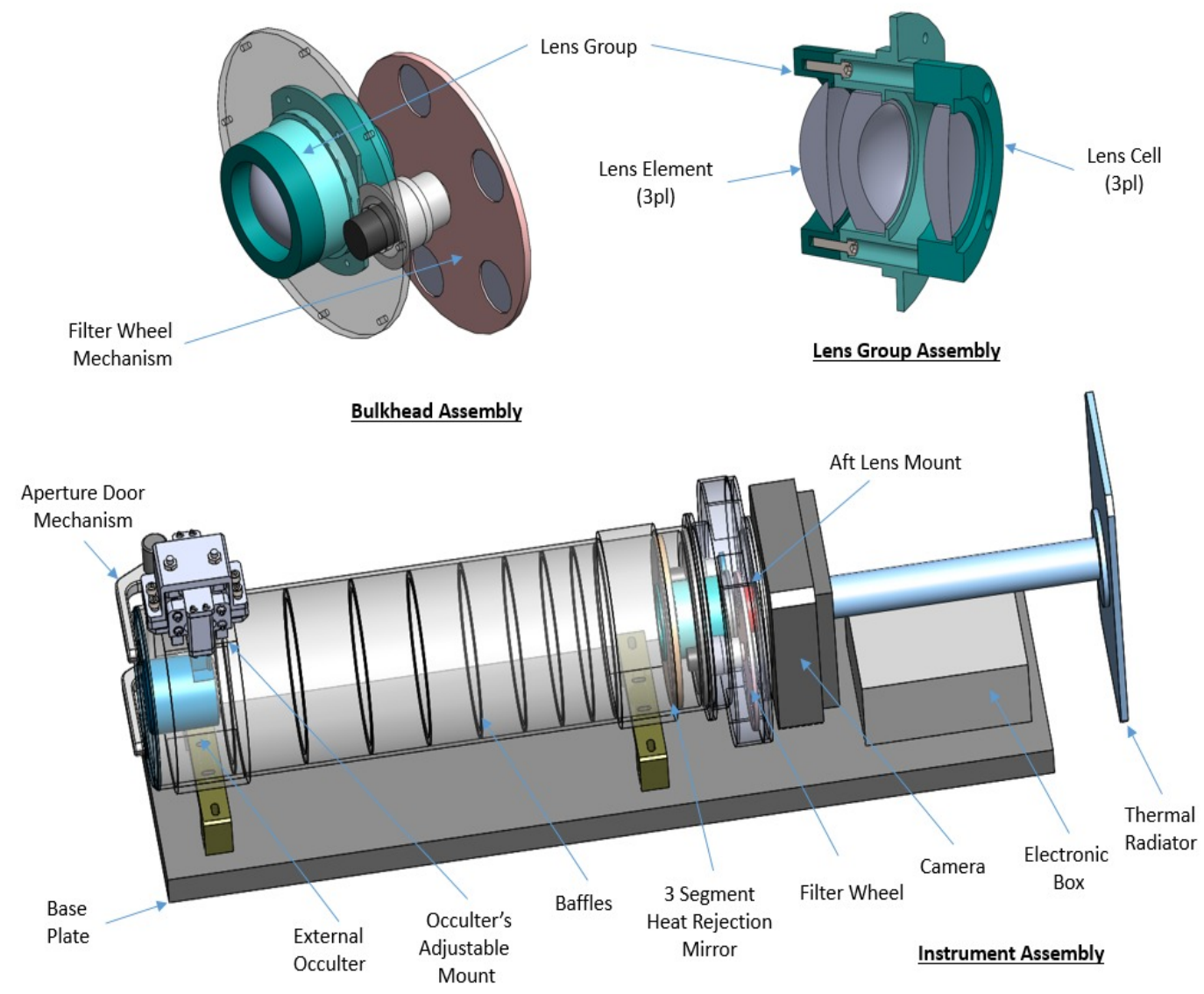

Figure 5. Mechanical design of the proposed miniature coronagraph to be used in BITSE. The bulkhead assembly, the lens group assembly, and the overall instrument assembly are shown.

\subsection{GMC mechanical design}

The GMC design consists of the following primary components: a modular aluminum structure, external occulter, aperture door mechanism, lens group, filter wheel, a polarization camera, thermal radiator, heat rejection mirror, base 


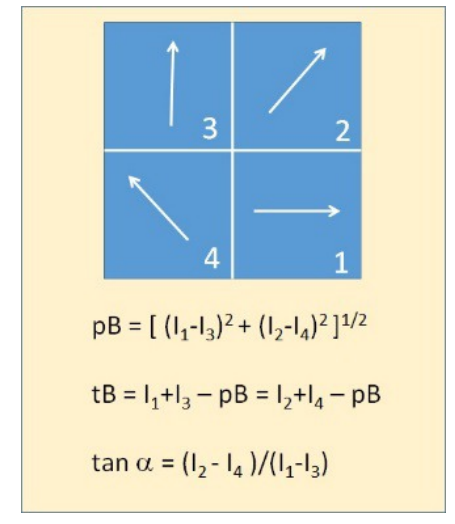

Proc. of SPIE Vol. 10769 107690X-6 Downloaded From: https://www.spiedigitallibrary.org/conference-proceedings-of-spie on 2/25/2019
Terms of Use: https://www.spiedigitallibrary.org/terms-of-use 
these four different orientations and the pattern repeats throughout the array. Combining the signals in four neighboring pixels, one gets a super pixel that gives both polarization (pB) and total (tB) brightness as described in Billings ${ }^{13}$. The entire array, therefore, gives the desired polarization and total brightness in a single exposure, albeit half the resolution. The polarization camera was recently tested in the laboratory ${ }^{14}$ and during the total solar eclipse of 2017 August 21 in the United States of America ${ }^{15}$. The tested camera has a $2048 \times 2048$-pixel monochrome CCD with $7.4 \mu \mathrm{m}$ square pixels. The effective size becomes $1950 \times 1950$ because of the mounting of the micropolarizer array on the CCD. Although the raw images have this size, $\mathrm{tB}$ and $\mathrm{pB}$ images will have a size of $950 \times 950$ super pixels due to combining four neighboring pixels. The resulting spatial resolution is more than adequate to study the larger-scale structures in the solar wind acceleration region. The exposure time can be set anywhere between $12 \mu \mathrm{s}$ and $16 \mathrm{~s}$. For the GMC mission applications, the exposure time will be $\geq 1 \mathrm{~s}$, well within the range. The CCD spectral response is in the range 300 - 1000 $\mathrm{nm}$, covering the wavelength range of the GMC mission. Figure 6 shows the way the neighboring pixels are combined to obtain tB and pB and the filter ratio images obtained during the 2017 August 21 total solar eclipse.
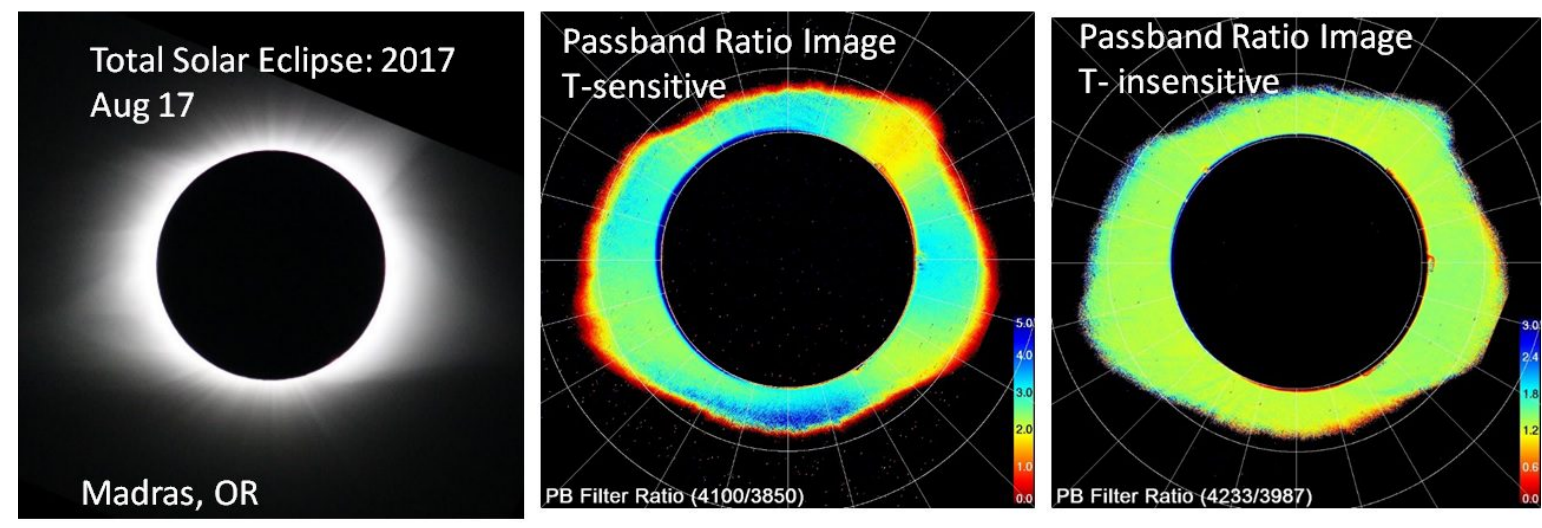

Figure 7. (left) White-light image of the 2017 August 21 total solar eclipse obtained using a Canon camera. Passband ratio images (4100/3850, middle) and (4233/3987, right) obtained using the polarization camera mounted on an F/8 Meade telescope. The temperature changes around the Sun is obvious in the 4100/3850 ratio image. The 4233/3987 ratio image is uniform because the solar wind flow has not picked up significantly close to the Sun.

Figure 7 shows the coronal during the eclipse and the passband ratio images taken using the polarization camera. The 4100/3850 ratio image demonstrates that the temperature variation in the corona is well-captured around the Sun. The $4233 / 3987$ image is uniform in the corona because the solar wind flow has not picked up in the corona close to the Sun ( $<1.5$ Rs imaged during the eclipse). Unlike the camera used for the eclipse observations future versions being developed at the University of Arizona (C. d'Aubigny, private communication) will be vacuum compatible for space flights.

\section{GMC MISSION}

The GMC spacecraft will be built using cubesat material deriving many aspects from the Dellingr CubeSat. The GMC mission will point the coronagraph to the Sun, obtain images at multiple wavelengths, and bring the data down to do science. Thus a 3-axis stabilized platform with a pointing accuracy of $\sim 1$ pixel is needed. Appropriate orbit needs to be chosen to maximize the time the coronagraph is pointed at the Sun. The GMC's length of $0.6 \mathrm{~m}$ and the telescope tube diameter of $\sim 12 \mathrm{~cm}$ requires a larger volume. Three 27-U Cubesat volumes arranged end-to-end would result in $\sim 30 \mathrm{x}$ 30 x $90 \mathrm{~cm}$ (81-U) CubeSat(http://www.planetarysys.com/web/wp-content/uploads/2014/08/2002367B-Payload-Specfor-3U-6U-12U-27U.pdf) with enough room for the GMC Instrument (see, e.g.,. Such a size would also be compatible with various launch options. The estimated mass of the coronagraph is $\sim 15 \mathrm{~kg}$ and the average power requirement is $\sim 20$ $\mathrm{W}$. These requirements can be readily accommodated in a SmallSat with a wet mass of $\sim 100 \mathrm{~kg}$.

\subsection{Mission architecture}

Figure 8 shows the architecture of the GMC mission. The onboard computer has the Attitude Determination and Control System (ADCS) module and the Command and Data Handling (C\&DH) module. The coarse Sun sensor (CSS), fine Sun sensor, star tracker, magnetometer, GPS antenna, torquers, and the reaction wheels are used to achieve the required 

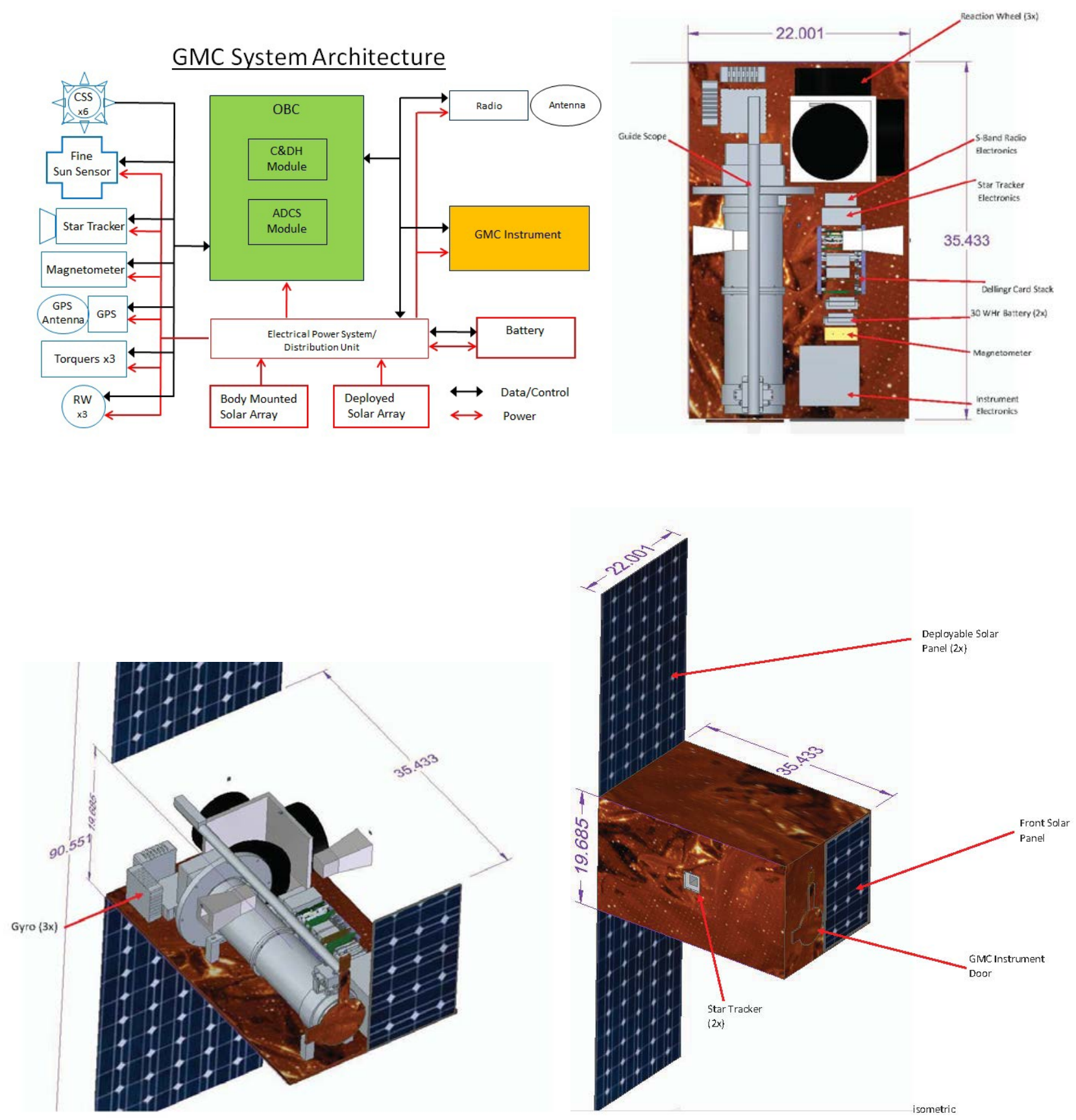

Proc. of SPIE Vol. 10769 107690X-8

Downloaded From: https://www.spiedigitallibrary.org/conference-proceedings-of-spie on 2/25/2019 Terms of Use: https://www.spiedigitallibrary.org/terms-of-use 


\subsection{Operations}

The Near Earth Network (NEN) project (https:/www.nasa.gov/directorates/heo/scan/services/networks/nen) at NASA/GSFC Wallops Flight Facility provides telemetry, commanding, ground-based tracking, data and communications services to a wide range of customers including those with satellites in Low Earth Orbit. The project will also manage mission operations. The Near Earth Network utilizes both NASA-owned and commercial stations around the world, so the large data volume can be readily handled. However, this means the data will be transmitted using S-band radio because the data volume is rather large ( 21 GB per day) depending on the cadence required. While the UHF/VHF radio communication is free, the S-band communication involves additional cost. According to the Small Spacecraft Technology State of the Art (NASA/TP-2015-216648/REV1) report from NASA/Ames there are many commercial antennas available that are compatible with S-band transmitters. Many companies that traditionally design communications for larger spacecraft modify some of their products for use on smaller spacecraft. These products have Technology Readiness Levels in the range 7-9 and hence will be used for the GMC mission.

\subsection{Launch and orbit options}

The candidate orbit choices for GMC were determined primarily by the expected launch opportunities. These included possible rideshare opportunities on Sun-synchronous launches and a simple release from the ISS. Since there was a preference to eliminate the need for a propulsion system, orbit lifetime analysis was performed to determine if an initial orbit altitude exists that satisfies the constraints defined by a desired 5-year minimum orbit lifetime and the requirement for the spacecraft to re-enter within 25 years of end-of-mission or 30 years of launch, accounting for the anticipated spacecraft ballistic properties, 3 sigma launch dispersions, and 2 sigma Solar flux predictions. It was assumed that a Pegasus launch vehicle would be used in order to obtain a worst-case estimate, as it has the largest launch dispersions of the vehicles being considered. Unfortunately, due to the wide variation in the launch dispersions and the Solar flux predictions, no orbit altitude exists that satisfies both orbit lifetime constraints without the aid of propulsion.

Analysis was also performed to estimate the delta-V necessary to maintain an ISS-like orbit of approximately $400 \mathrm{~km}$ over the desired 5-year mission lifetime. Using worst-case estimates of ballistic properties and solar activity, it was found that the required delta- $\mathrm{V}$ would be approximately $300 \mathrm{~m} / \mathrm{s}$. Given a total spacecraft initial mass estimate of 125 $\mathrm{kg}$, and assuming a specific impulse of $220 \mathrm{~s}$, the propellant mass required would be approximately $16 \mathrm{~kg}$. Table 2 summarizes various options including the pros and cons. Clearly, the dawn-to-dusk Sun synchronous orbit is preferable because the Sun can be observed continuously. However, this orbit needs some propulsion and the number of launch opportunities may be limited. The 700-km altitude polar orbit without propulsion can last for $\sim 5$ years without propulsion, but the Sun can be observed only for half the orbit. The ISS orbit has the problem of short lifetime ( 1 year) compounded by the shorter observing time.

Table 2. Orbit options for the GMC mission

\begin{tabular}{|l|l|l|}
\hline Orbit Description & Pros & Cons \\
\hline $\begin{array}{l}\sim 700 \mathrm{~km} \text { altitude Polar Orbit } \\
\text { without Propulsion }\end{array}$ & $\begin{array}{l}>5 \text { years before orbit decay, } \\
\text { multiple rideshare opportunities }\end{array}$ & Only see Sun for $\sim$ half an orbit \\
\hline $\begin{array}{l}\sim 400 \mathrm{~km} \text { altitude, ISS Orbit } \\
\text { without propulsion }\end{array}$ & $\begin{array}{l}\text { Lowest launch cost and most } \\
\text { launch opportunities }\end{array}$ & $\begin{array}{l}\text { Only see Sun for } \text { half an orbit; only } \\
\text { to 2 years before orbit decays }\end{array}$ \\
\hline $\begin{array}{l}\sim 700 \mathrm{~km} \text { altitude, 6AM-6PM } \\
\text { Propulsion }\end{array}$ & $\begin{array}{l}\text { Almost continuous viewing of } \\
\text { the Sun }\end{array}$ & $\begin{array}{l}\text { Requires propulsion; fewer rideshare } \\
\text { opportunities (possibly dedicated } \\
\text { launch vehicle) }\end{array}$ \\
\hline $\begin{array}{l}\sim 400 \mathrm{~km} \text { altitude ISS Orbit with } \\
\text { propulsion }\end{array}$ & $\begin{array}{l}\text { Lowest launch cost and most } \\
\text { launch opportunities }\end{array}$ & $\begin{array}{l}\text { Only see the Sun for } ~ \text { half an orbit, } \\
\text { possible ISS safety issues with } \\
\text { propulsion system }\end{array}$ \\
\hline
\end{tabular}


The re-entry requirements can be significantly relaxed with the new technology to enhance drag to induce re-entry over a short period of time (http://www.tethers.com/TT.html) using the terminator tether. The tether needs to be attached to the spacecraft before launch and released when deorbiting is desired. The 5-km long tether interacts with the ionospheric plasma and the current induced in the tether results in additional drag. Over a period of weeks to months, the spacecraft re-enters and the tether burns up with the spacecraft.

As for the launch options, we considered three options: (i) The Pegasus air-launch system can be readily used and has launched many small satellites. (ii) The Space Station Integrated Kinetic Launcher for Orbital Payload Systems (SSIKLOPS), commonly referred to as Cyclops. (iii) Spaceflight Secondary Payload System (SHERPA).

The Pegasus air-launch involves a dedicated launch vehicle. The Cyclops launch has the following constraints on the satellites $^{16}$ : (i) total wet mass up to $\sim 100 \mathrm{~kg}$ and (ii) a payload envelope of up to $\sim 0.9 \times 0.8 \times 0.5 \mathrm{~m}$ (rectangular box). Both these constraints can be readily met with the wet mass $(\sim 100 \mathrm{~kg})$ and volume (see Fig. 9$)$ of the GMC mission. Cyclops will operate from the Japanese Engineering Module (JEM). It will make use of the existing slide table in JEM's airlock. The satellite is attached to Cyclops, which is placed onto the airlock slide table and processed through to the external environment. Cyclops is then grasped by the robotic arm from which Cyclops deploys the satellite. In the case of SHERPA, adapter selection is wet-mass limited. With a 100-kg wet mass of the GMC spacecraft, the Vertical Port Adapter is the appropriate interface (www.spaceflight.com/wp-content/uploads/2015/05/SPUG-RevF.pdf).

A recent development provides another opportunity to boost the GMC spacecraft orbit above the ISS orbit. An Orbital ATK Cygnus cargo spacecraft, after departing ISS, boosted its orbit to $500 \mathrm{~km}$ and deployed four satellites (https://spacenews.com/spire-deploys-four-satellites-from-cygnus/). The 500-km orbit extends the life of CubeSats by a factor of 2 and hence is a viable alternative to Cyclops launch.

\section{DISCUSSION AND SUMMARY}

There is a scientific need to operate the coronagraph for many years to provide useful data to study the physical properties of the steady and transient flows in the corona. One of the major concerns in using CubeSats (or structures based on CubeSat material in our case) is their short lifetime due to radiation. With recent developments in material that can withstand radiation, it is possible to enhance the lifetime of CubeSats and the SmallSats such as the GMC spacecraft. The NASA Langley Research Center has developed lightweight radiation shielding (https://technology.nasa.gov/patent/LAR-TOPS-250) by layering high atomic number metals such as tantalum on aluminum sheets that can be formed into standardized enclosures for CubeSats and SmallSats. This technology provides a low-cost means to protect CubeSat electronics from ionizing radiation found in Low Earth Orbits. Estimates have shown that the Z-shield approach can boost the life of cubesat electronics by an order of magnitude.

To summarize, we presented a SmallSat mission featuring the Goddard Miniature Coronagraph (GMC) that can be deployed in the ISS orbit or a polar orbit to provide images of the solar corona. GMC is a single-stage coronagraph with a tapered, hollow right frustum with a threaded surface as its external occulter. The hollow-cone occulter designed and manufactured at NASA/GSFC has shown a superior diffraction-reduction capability, better than that of a multi-disk occulter used in traditional coronagraphs. A version of the GMC assembly has been tested in the laboratory and in the NCAR vacuum tank facility in Boulder. Optical and mechanical designs have been refined and ready for building a prototype. The coronagraph will utilize a polarization camera, which eliminates the need for a polarization wheel normally employed in traditional coronagraphs. The polarization camera has a polarization mask overlaid on the face of the CCD such that four consecutive pixels receive light with different polarization orientation. Summing four neighboring pixels yields total and polarized brightness of the solar corona. Currently the polarization camera is not vacuum compatible, but efforts are underway to make it ready for space flight. GMC will be accommodated in a small satellite that can be built with cubesat material to minimize the cost. The overall size of the current spacecraft is $50 \mathrm{~cm} \times$ $56 \mathrm{~cm} \times 90 \mathrm{~cm}$. Deployed and body-mounted solar panels can satisfy all the power requirements of the mission. The radio communication will be done using S-band because the UHF/VHF radio cannot handle the large data volume generated by the coronagraph. 


\section{ACKNOWLEDGMENTS}

We thank P. Nikulla, L. Purves, F. Vaughn, C. Clagett, S. West, N. Reginald, S. Yashiro, and P. Mäkelä for their contributions to this study. The 2017 August 21 eclipse expedition was supported by NASA. Work supported by NASA/GSFC Internal Research and Development (IRAD) funds.

\section{REFERENCES}

[1]. Brueckner, G. E. et al., “The Large Angle Spectroscopic Coronagraph (LASCO)”, Solar Phys. 162, 357 (1995).

[2]. Howard, R. A. et al., "Sun Earth Connection Coronal and Heliospheric Investigation （SECCHI)", Space Sci. Rev. 136, 67 (2008).

[3]. National Academies of Sciences, Engineering, and Medicine, “Achieving Science with CubeSats: Thinking Inside the Box”. The National Academies Press. Washington, DC. (2016), doi:10.17226/23503.

[4]. Cram, L. E., "Determination of the temperature of the solar corona from the spectrum of the electron-scattering continuum”, Solar Phys. 48, 3 (1976).

[5]. Ichimoto, K., Kumagai, K., Sano, I., Kobiki, T., Munoz, A., Sakurai, T., "Measurement of the Coronal Electron Temperature at the Total Solar Eclipse on 1994 November 3”, PASJ 48, 285 (1996).

[6]. Takahashi, N. Yoneshima, W., Hie, E., "Large-Scale Distribution of Coronal Temperature Observed at the Total Solar Eclipse on 26 February 1998”, ASP Conf. Ser. 205, 121 (2000).

[7]. Reginald, N. L., St. Cyr, O. C., Davila, J. M., and Brosius, J. W., "Electron Temperature and Speed Measurements in the Low Solar Corona: Results From the 2001 June Eclipse”, Astrophys. J. 599, 596 (2003).

[8]. Reginald, N. L. and Davila, J. M. "MACS for Global measurement of the Solar wind velocity and the Thermal electron temperature during the Total solar eclipse on 11 August 1999”, Solar Phys. 195, 111 (2000).

[9]. Gong, Q. and Socker, D., "Theoretical study of the occulted solar coronagraph, Optical Systems Degradation, Contamination, and Stray Light: Effects, Measurements, and Control”, Proc. SPIE 5526, 208-219 (2004).

[10]. Bout, M., Lamy, P., Maucherat, A., Colin, C., Llebaria, A., "Experimental Study of External Occulters for the Large Angle and Spectrometric Coronagraph 2: LASCO-C2”, Applied Optics 39, 3955-3962 (2000).

[11]. Thernisien, A., Colaninno, R. C., Plunkett, S., Socker, D. G., Gong, Q., Landini, F., "Experimental and numerical optimization of a coronagraph external occulter. Application to SECCHI-COR2 and GOES-R SCOR”, Proc. SPIE 5901, 366-376 (2005).

[12]. Brock, N. J., Crandall, C., and Millerd, J. E., "Snap-shot imaging polarimeter: Performance and applications," Proc. SPIE 9099, pp. 9099031-90990312 (2014).

[13]. Billings, D. E., [A guide to the solar corona], Academic Press, New York, (1966).

[14]. Reginald, N. L., Gopalswamy, N., Yashiro, S., Guhathakurta M., "Replacing the polarizer wheel with a polarization camera to increase the temporal resolution and reduce the overall complexity of a solar coronagraph", JATIS, 3, id. 014001 (2017).

[15]. Gopalswamy, N., Yashiro, S., Reginald, N., Thakur, N., Thompson, B. J., Gong, Q., "Physical Conditions in the Solar Corona Derived from the Total Solar Eclipse Observations obtained on 2017 August 21 Using a Polarization Camera”, American Astronomical Society, AAS Meeting \#231, id. 220.08 (2018).

[16]. Newswander, D. R., Smith, J. P., Lamb, C. R., and Ballard, B. G., "Space Station Integrated Kinetic Launcher for Orbital Payload Systems (SSIKLOPS) - Cylops”, Proc. 27th Annual AIAA/USU Conference on Small Satellites, SSC-13-V-2, (2013). 